\title{
Randomised controlled trial of a postpartum relapse prevention intervention
}

\author{
Susan M Van't Hof, Michael A Wall, David W Dowler, Michael J Stark
}

Many women quit smoking during pregnancy but postnatal relapse rates are high, averaging $50-80 \%$ in the first year after delivery. ${ }^{12}$ Previous work suggests that provider based relapse intervention in the context of well-baby visits may lead to a decrease in postnatal relapse rates. ${ }^{3}$ However, prior research also suggests that the majority of postnatal providers do not take a systematic approach to obtaining a smoking history from all new mothers, and thus may miss opportunities for cessation and relapse counselling. ${ }^{34}$

In the present study we examined whether: (1) a relapse prevention intervention, implemented during the hospital stay during the period soon after delivery and at well-baby visits, would reduce the rate of relapse to smoking six months postpartum; (2) the time of delivery was an opportune moment to obtain a smoking history; (3) the history could be transmitted quickly to the infant's pediatric provider; and (4) transmission would lead to increased rates of relapse advice.

\section{Methods}

All women delivering babies at six participating Portland, Oregon, metropolitan area hospitals received an in-hospital screening and were deemed eligible for the study if they reported smoking during the 30 days before the pregnancy and quitting during pregnancy, and were willing to speak with a Visiting Nurse Association (VNA) nurse about having quit smoking. Women were not eligible to be screened if there was a maternal or child illness that would prevent them from attending the paediatric well-baby visits; if the baby was being adopted; or if the woman did not speak English.

When an eligible woman agreed to participate in the study, the delivery nurse or the birth certificate clerk contacted the VNA. A VNA nurse informed the woman about the study, obtained informed consent, collected a saliva sample for cotinine verification of non-smoking status, and conducted the baseline assessment interview, preferably while the participant was still in the hospital. However, because of short postpartum hospital stays, just over half of the participants were interviewed in the hospital. The average time to interview for those interviewed in the hospital was 1.5 days postpartum (range $0-8$ days), while the interview took place an average of 8.9 days postpartum (range 2-28 days) for those seen after discharge.

The baseline interview took approximately 20-30 minutes. Upon completion of the interview, the participant was randomised into either a control or relapse prevention intervention group. Controls received no intervention from the VNA nurse, and standard care from their paediatric provider. Women in the intervention group received a 15-30 minute relapse prevention intervention from the VNA nurse which included counselling about reasons for maintaining cessation and help in developing a plan for doing so. At the two week, and two and four month well-baby visits with the paediatric provider they received reinforcement if they had done so and if not, given encouragement and a plan to try to quit again.

After the VNA intervention, the principal investigator (PI) called all of the paediatric providers who would be seeing participants in the intervention group. All of the 85 paediatric providers contacted in this manner agreed to participate in the study. During this initial call, the PI conveyed the maternal smoking history, explained the purpose of the study, and briefly discussed how to conduct relapse prevention in the context of well-baby care. For subsequent participants who would be seeing the same paediatric provider, a letter with the participant's and infant's names, and a reminder about the study was faxed from the PI to the paediatric provider. The call or letter from the PI was followed by a packet of information that included a letter to the provider, chart stickers, tips on relapse prevention, and written materials for the participant.

Of the 9390 women who delivered and were eligible to be screened during the 14 months of recruitment, 9140 (97\%) were successfully screened. Of the 1837 (20\%) new mothers who reported smoking cigarettes 30 days before pregnancy, 638 (35\%) reported to have quit smoking during their pregnancy or within 30 days of becoming pregnant. Only 287 $(55 \%)$ of those eligible were enrolled in the study; 141 were randomised into the experimental group, 146 into the control group. The others either refused to participate (196), relapsed back to smoking before the baseline interview (27), or were otherwise unable to enroll for a variety of reasons (12). Two women were excluded from the study because their cotinine concentrations indicated recent smoking.

Baseline data were collected during a face to face interview at the time of initial enrolment in the study. A follow up telephone interview was conducted approximately six months postpartum. Four women were excluded from the study at follow up because they were moving out of the Portland metropolitan area or were in the military, and thus had no specific 
Table 1 Participant characteristics, by group assignment

\begin{tabular}{llll}
\hline & $\begin{array}{l}\text { Control } \\
(n=144)\end{array}$ & $\begin{array}{l}\text { Intervention } \\
(n=133)\end{array}$ & Total \\
\hline White & $75 \%$ & $85 \%$ & $80 \%$ \\
Married or living with partner & $67 \%$ & $62 \%$ & $64 \%$ \\
Completed high school & $65 \%$ & $64 \%$ & $65 \%$ \\
Completed college & $10 \%$ & $7 \%$ & $8 \%$ \\
Birth was first live birth after 20 weeks & $62 \%$ & $61 \%$ & $61 \%$ \\
Mean (SD) age & $25.2(6.0)$ & $24.0(5.3)$ & $24.6(5.7)$ \\
Mean (SD) number of cigarettes smoked per & $11.8(8.0)$ & $12.5(8.2)$ & $12.1(8.1)$ \\
$\quad$ day before pregnancy & $43 \%$ & $50 \%$ & $46 \%$ \\
Husband/partner smokes & $64 \%$ & $66 \%$ & $65 \%$ \\
Very confident to remain a non-smoker & & & \\
$\star$ & & &
\end{tabular}

paediatric provider during the follow up period; two women were excluded because they were not keeping their babies; and four did not complete the follow up survey, yielding 277 who completed the study.

Baseline and follow up measures were analysed using logistic regression. The primary dependent variable was smoking status at follow up, defined as any smoking, even a puff, in the previous seven days. Independent variables of interest included group assignment (intervention or control), demographics (race, age, education, employment, children, living with partner), and smoking related variables (age first smoked, number daily cigarettes smoked before quitting, time until first cigarette of the day, partners', friends' and family members' smoking status, number smokers in the home, home smoking rules, family, friends, and co-workers support for not smoking, confidence to stay quit, and depressive symptoms).

Bivariate logistic regression was used to establish which independent variables would be tested in the multivariate model (cut off for inclusion $\mathrm{p}<0.10$ ). The final multivariate model was built with the variables that retained significant independent associations with relapse status $(\mathrm{p}<0.05)$.

Correlation and contingency table analyses were used to investigate whether reports of doctor advice to quit and receipt of relapse prevention materials were greater for women in the experimental group.

\section{Results}

Table 1 displays the background characteristics of the women in the sample. Overall the group was predominantly white, which is consistent with the demographic profile of people in the Portland area. A significant relationship emerged between race and group assignment, as cases in the control group were more likely to be non-white. The women from the control

Table 2 Logistic regression analysis: variables associated with relapse $(n=270)$

\begin{tabular}{llll}
\hline Variable & Variable level & \% Relapsed & OR (95\% CI) \\
\hline Confidence to stay quit & $\begin{array}{l}\text { Very confident } \\
\text { None/a little/somewhat } \\
\text { confident }\end{array}$ & 32 & 1.00 \\
& More encouragement & 25 & $1.81(1.05$ to 3.12) \\
Family and friends' & Less encouragement & 51 & 2.00 \\
encouragement to stay quit & None & 17 & 1.00 \\
Number of friends/ family who & A few & 38 & $3.49(1.25$ to 9.78$)$ \\
smoke & Most/all & 49 & $5.33(1.85$ to 15.40$)$ \\
\hline
\end{tabular}

OR, odds ratio; CI, confidence interval group and intervention group were similar across all other baseline demographic and smoking related characteristics.

Women in the experimental group were more likely to report that a doctor or nurse talked with them about smoking at least once since delivery $(71 \% v 20 \%$ of control group: $\left.\chi^{2}=73.56, p<0.001\right)$. Furthermore, a significant and strong association emerged between experimental assignment and total number of times the women reported a provider talked about staying quit (control group mean $=0.4$, intervention group mean $=2.0 ; \mathrm{F}=63.14$, $\mathrm{p}<0.001)$. Women in the intervention group were also more likely to report receiving written materials about how to stay quit (47\% $v 3 \%$ of the control group; $\chi^{2}=73.21$, $\mathrm{p}<0.001)$.

There was no difference in the relapse rate between women in the intervention $(41 \%)$ and control $(37 \%)$ groups. Even when all those lost to follow up were considered to have relapsed, differences between intervention (42\%) and control (38\%) groups did not vary significantly.

In an examination of the variables that were related to relapse, the final multivariate model suggested independent associations between relapse and a handful of predictor variables. Factors found to have a significant and independent association with smoking relapse are specified in table 2 .

Table 2 shows that relapse rates were significantly higher among women who had lower confidence for maintaining cessation, less encouragement from family and friends to refrain from smoking, and a greater number of smokers among their family and friends.

Posthoc analyses were done to investigate intervention effects on smoking related outcomes other than smoking rate. No significant associations were found between experimental group assignment and: (1) days until relapse; (2) time before first cigarette after waking; (3) number of quit attempts since delivering the baby; and (4) number of cigarettes smoked per day.

\section{Discussion}

Women in this study had quit smoking during pregnancy but had a high postpartum relapse rate, indicating the need for effective relapse prevention interventions to protect new mothers and babies against the ill effects of smoking. Our results show that paediatric providers will deliver relapse prevention messages to infants' mothers if they are informed that the mothers quit smoking during pregnancy. Despite our success in getting providers to deliver the relapse prevention intervention, the intervention itself was insufficient to reduce relapse. Despite the difference in methodology offered by our study through a face to face intervention, our findings were consistent with prior research on smoking relapse prevention among pregnant women.

We found a moderate association between confidence to stay quit and non-relapse. The effectiveness of provider delivered messages may be increased if they go beyond risk 
communication and attempt to bolster women's confidence in their ability to stay quit. Our findings also show that postpartum relapse was strongly associated with both the presence of smokers among participants' family and friends, and the absence of encouragement from family and friends to stay quit. Effective relapse prevention may need to employ ancillary staff to intervene with women's social networks in support of new mothers' efforts to stay quit.

We found that the hospital stay around the time of delivery may be an opportune time to obtain a woman's smoking history. We obtained smoking history from $97 \%$ of the eligible women, and the smoking rate of $20 \%$ and the quit rate of $35 \%$ are within the expected range. ${ }^{36}$ However, since $45 \%$ of those eligible refused participation in the study, the time around delivery may not be opportune for recruiting women into a study. The high refusal rate may also reflect the possibilities that women who have truly quit may perceive no need to be in a study, and that some women intend to resume smoking after delivery and may not wish to be involved in a relapse prevention study.
In sum, we found that communicating a new mother's smoking status from the delivery service to the infant's paediatric provider can be done quickly and effectively, and leads to increased rates of provider delivered smoking relapse prevention advice. We suggest that for pregnant smokers, relapse prevention should: (1) be added to cessation interventions; (2) include messages aimed at bolstering confidence in staying quit; (3) be delivered during prenatal care and postpartum through the infant's paediatric provider; and (4) be presented to both the woman and her social network by ancillary staff.

1 Ockene J. Smoking among women across the life span: prevalence, interventions, and implications for cessation research. Ann Behav Med 1993;15:135-48.

2 Fingerhut L, Kleinman J, Kendrick J. Smoking before, during, and after pregnancy. Am F Public Health 1990;80:5415 .

3 Wall MA, Severson HH, Andrews JA, et al. Pediatric officebased smoking intervention: impact on maternal smoking and relapse. Pediatrics 1995;96:622-8.

4 Frankowski BL, Weaver SO, Secker-Walker RH. Advising parents to stop smoking: pediatricians' and parents'
attitudes. Pediatrics 1993;91:296-300.

5 McBride CM, Curry SJ, Lando HA, et al. Prevention of McBride CM, Curry SJ, Lando HA, et al. Prevention of
relapse in women who quit smoking during pregnancy. $\mathrm{Am}$ relapse in women who quit smoking
f Public Health 1999;89:706-11.

6 McBride CM, Pirie PL. Postpartum smoking relapse. Addict Behav 1990;15:165-8. 